\title{
Dark clouds in the vicinity of the emission nebula Sh2-205: interstellar extinction and distances ${ }^{\star}$
}

\author{
V. Straižys ${ }^{1}$, V. Čepas ${ }^{1}$, R. P. Boyle ${ }^{2}$, J. Zdanavičius ${ }^{1}$, M. Maskoliūnas ${ }^{1}$, A. Kazlauskas ${ }^{1}$, \\ K. Zdanavičius ${ }^{1}$, and K. Černis ${ }^{1}$
}

\author{
${ }^{1}$ Institute of Theoretical Physics and Astronomy, Vilnius University, Sauletekio al. 3, 10222 Vilnius, Lithuania \\ e-mail: vytautas.straizys@tfai.vu.1t \\ 2 Vatican Observatory Research Group, Steward Observatory, Tucson, AZ 85721, USA
}

Received 14 December 2015 / Accepted 8 March 2016

\begin{abstract}
Results of CCD photometry in the seven-colour Vilnius system for 922 stars down to $V=16-17$ mag and for 302 stars down to 19.5 mag are used to investigate the interstellar extinction in an area of 1.5 square degrees in the direction of the P7 and P8 clumps of the dark cloud TGU H942, which lies in the vicinity of the emission nebula Sh2-205. In addition, we used 662 red clump giants that were identified by combining the 2MASS and WISE infrared surveys. The resulting plots of extinction versus distance were compared with previous results of the distribution and radial velocities of $\mathrm{CO}$ clouds and with dust maps in different passbands of the IRAS and WISE orbiting observatories. A possible distance of the front edge of the nearest cloud layer at $130 \pm 10 \mathrm{pc}$ was found. This dust layer probably covers all the investigated area, which results in extinction of up to 1.8 mag in some directions. A second rise of the extinction seems to be present at 500-600 pc. Within this layer, the clumps P7 and P8 of the dust cloud TGU H942, the Sh2-205 emission nebula, and the infrared cluster FSR 655 are probably located. In the direction of these clouds, we identified 88 young stellar objects and a new infrared cluster.
\end{abstract}

Key words. open clusters and associations: general - stars: fundamental parameters -ISM: individual objects: TGU H942 P7 ISM: clouds - ISM: individual objects: TGU H942 P8

\section{Introduction}

The emission nebula Sh2-205 (Sharpless 1959) is the brightest northern clump of a large H II region at the border of Perseus and Camelopardalis having a size of $3.5^{\circ} \times 4^{\circ}$. The star HD 24431 of spectral class O9 ( $V=6.7-6.8 \mathrm{mag})$, located close to its centre, is considered as the main ionising source of the nebula. The strongest emission spots in $\mathrm{H} \alpha$ are observed around HD 24431 (the nebula [GS55] 34, Gaze \& Shain 1955) and in the northern clump (Sh2-205). It is doubtful that HD 24431 is the only ionising star of this huge group of nebulae. Since no more O-type stars are known in the vicinity, the following early-B type stars have been considered: HD 23675 (B0 III), ALS 7793 (B1 V), and HD 24094 (B1 III), see Avedisova \& Kondratenko (1984), Foster \& MacWilliams (2006), Romero \& Cappa (2008). The last star is at the centre of the nebula Sh2-205, its spectral type is B1 III (Bouigue 1959), B2 $\tau$ (Rydström 1978), B1 IV (Zdanavičius et al. 2001), and B1 V (De Cat et al. 2007) ${ }^{1}$. The outstanding spectral classes B8 and B6 are given in the HD catalogue and in Johnson (1956), respectively. In these cases the star might be misclassified because of interstellar Ca II lines, or its spectrum might be variable. The star is known to have variable brightness; in the General Catalogue of Variable Stars its name is CY Cam, the amplitude is about $0.1 \mathrm{mag}$, and the period is $1.05 \mathrm{~d}$. It can be either a $\beta$ Cephei pulsating

\footnotetext{
* Full Tables 1 and 2 are only available at the CDS via anonymous ftp to cdsarc.u-strasbg. fr (130.79.128.5) or via

http://cdsarc.u-strasbg.fr/viz-bin/qcat?]/A+A/590/A21

1 The last two spectral types are determined from multi-colour photometry in the Vilnius and Geneva photometric systems, respectively.
}

star or a contact eclipsing binary of type EW (Duerbeck 1997; De Cat et al. 2007).

Distances to the Sh2-205 nebula and its southern extension, [GS55] 34, are known with a low accuracy. All the authors agree that HD 24431 is of spectral class O9, but the luminosity class of the star is rather indefinite and ranges from V (Conti \& Alschuler 1971) to IV-V (Morgan et al. 1953) and III (Sota et al. 2011). This places the star (and the nebula) somewhere between 650 and $950 \mathrm{pc}$ from the Sun. The star is a close binary (WDS $\mathrm{J} 03556+5238 \mathrm{AB}, \Delta m=3 \mathrm{mag}$, sep $=0.7^{\prime \prime}$ ), and this might be the reason of luminosity errors. Kinematic distances to the nebulae, determined from radial velocities of $\mathrm{H} \alpha, \mathrm{HI}$, or $\mathrm{CO}$ lines in the related clouds and the rotation curve of the Galaxy, are even more contradictory (Blitz et al. 1982; Fich \& Blitz 1984; Fich et al. 1990; Foster \& MacWilliams 2006; Foster \& Brunt 2015).

In the vicinity of the nebula and to north-east of its northern part, a number of dark clouds are observed. In the atlas and catalogue of dark clouds published by Dobashi et al. (2005) they are called the P7 and P8 clumps of cloud TGU H942. These clumps are parts of a dust ring with a diameter of $8^{\circ}$ located at the edge of the Camelopardalis and Perseus constellations, and identified by Straižys \& Laugalys (2007a, 2008). Clump P8 is located between the Sh2-205 and [GS55] 34 nebulae, while clump P7 is located about $0.4^{\circ}$ north-east from the northern edge of Sh2205. Within these clumps and in their vicinities, Dobashi (2011) has identified 14 smaller dust clouds on the basis of reddening and extinction of stars present in the 2MASS catalogue. Most of these dust clouds (if not all) are probably located in the gas and 
dust complex to which the Sh2-205 nebula belongs. Thus, the investigation of distances to the dark clouds may help to find a more reliable distance to the nebula.

Photoelectric photometry of stars in the wide vicinity of Sh2-205 in the Vilnius seven-colour system has been started by Zdanavičius et al. (2001) and Zdanavičius \& Zdanavičius (2002) with the aim to determine distances to the Camelopardalis complex of dark clouds. The work was extended with CCD photometry by Čepas et al. (2013; hereafter Paper I) in a $1.5 \mathrm{deg}^{2}$ area with the centre at $\alpha(2000)=03^{\mathrm{h}} 57.0^{\mathrm{m}}, \delta(2000)=+53^{\circ} 40^{\prime}$; $\ell=148.34^{\circ}, b=+0.21^{\circ}$, including clump P7 and part of clump P8 of TGU H942. The area also covers the northern end of the Sh2-205 nebula. In this study $V$ magnitudes and six colour indices were measured for 922 stars down to $V \approx 17 \mathrm{mag}$. Using the photometric interstellar reddening-free parameters, the authors determined for most of these stars photometric spectral types in the MK system.

In a smaller area, we here extend the multi-colour photometry of stars and their two-dimensional classification down to $V \approx 19.5$ mag. The results of the present paper and Paper I are then used to investigate the interstellar extinction run with distance and to estimate distances to dark clouds in the area. To extend the extinction versus distance dependence to larger distances, we additionally considered red clump giants (RCGs) that we identified with the help of the 2MASS and WISE surveys. The same surveys are used to identify more young stellar objects (YSOs) in the area.

\section{Photometric data and spectral types}

As we mentioned in the Introduction, we here used the catalogue of 922 stars published in Paper I. CCD exposures of the investigated area (Fig. 1) in the Vilnius seven-colour system were obtained in October of 2010 with the Maksutov-type $35 / 51 \mathrm{~cm}$ telescope of the Molètai Observatory in Lithuania. More details about the observations and their processing are given in Paper I. The Vilnius photometric system UPXYZVS with the mean wavelengths 345, 374, 405, 466, 516, 544, and $656 \mathrm{~nm}$ has been described by Straižys (1992). For about $70 \%$ of the stars, two-dimensional spectral types (spectral and luminosity classes) were determined by applying two classification codes based on interstellar reddening-free $Q$-parameters. The classification codes use the interstellar reddening-free $Q$-parameters that are compared either to $Q$-parameters of individual standard stars with known MK classification (the code COMPAR) or to artificial stars with $Q$-parameters calculated from intrinsic colour indices calibrated in MK types (the code KLASQ). In the present investigation, the stars of Paper I were reclassified anew using a modernised version of the KLASQ code. To classify $K$ and $\mathrm{M}$ dwarfs, we also used the Vilnius diagram $Y-Z$ vs. $Z-V$ (see Zdanavičius et al. 2012). To identify $M$ dwarfs, we additionally applied the diagram $J-K_{\mathrm{s}}$ vs. $W 1-W 2$, where the first colour index is in the 2MASS system and the second in the WISE system. For some stars the new spectral and luminosity classes show small differences with Paper I because of the listed changes.

To classify fainter stars down to $V \approx 19.5 \mathrm{mag}$, we applied the Vilnius photometry in a $12^{\prime} \times 12^{\prime}$ area located in a region of intermediate transparency (Fig. 1) with the centre coordinates $\alpha(2000)=03^{\mathrm{h}} 58.9^{\mathrm{m}}, \delta(2000)=+53^{\circ} 39^{\prime} ; \ell=148.56^{\circ}, b=$ $+0.38^{\circ}$. CCD observations in the Vilnius system in this area were obtained in December of 2013 with the $1.8 \mathrm{~m}$ VATT telescope of the Vatican Observatory on Mt. Graham, Arizona. Twenty-five frames with exposures of $30 \mathrm{~min}$ to $6 \mathrm{~s}$ were processed with the

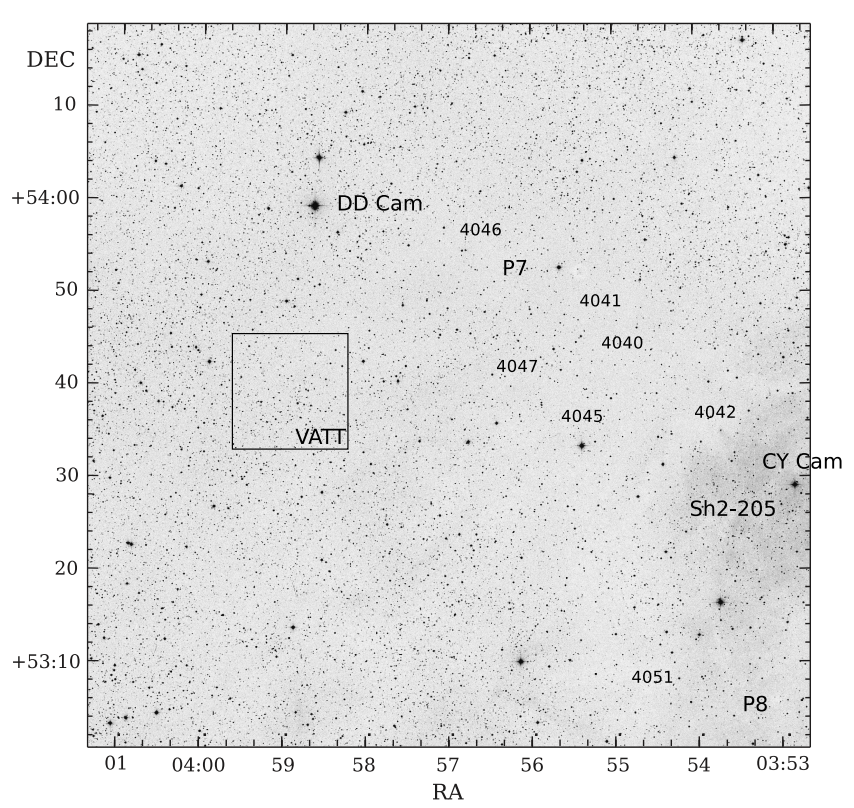

Fig. 1. Map with the observed area in the red filter (DSS1 Red from the SkyView Virtual Observatory). The small square shows the area observed with the VATT telescope to fainter magnitudes. Approximate positions of the northern part of the emission nebula Sh2-205, the centre of the dark cloud TGU H942 P7, and a part of the cloud TGU H942 P8 are labelled. The four-digit numbers designate positions of a few clouds that were identified by Dobashi (2011). The brightest star in the area is the eclipsing variable DD Cam (HD 24733). CY Cam might be the ionising star of Sh2-205.

IRAF program package in the aperture photometry mode. Instrumental colour indices were transformed to the standard system with colour equations obtained from observations of the cluster M67 (Laugalys et al. 2004). The stars measured in Paper I served as zero-point standards of magnitudes and colour indices. The final adjustment of zero-points was made by optimising the accuracy of the photometric classification of a selected set of standard stars in the investigated area. Photometric classification of stars was made with the same codes and diagrams mentioned above.

The catalogue of photometric data for 302 stars down to $V \approx$ $19.5 \mathrm{mag}$, and the results of the photometric two-dimensional classification for about $80 \%$ of them are given in Table 1 . The catalogue is accessible at the Strasbourg Data Center. We provide the beginning of Table 1 for the first five stars. The table contains the following information: star number, equatorial coordinates $\mathrm{J} 2000.0$, magnitude $V$, colour indices $U-V, P-V, X-V$, $Y-V, Z-V$ and $V-S$, and photometric spectral types in the MK system. Photometric spectral classes are given in lower-case letters to distinguish them from spectroscopic classes. The suspected metal-deficient stars are designated as "md:", and the metal-rich stars (Am) as "mr:". The coordinates are from the PPMXL catalogue (Roeser et al. 2010) rounded to two decimals of time second and to one decimal of arcsecond.

The accuracy of photometry in different ranges of magnitudes is approximately the same as in the cloud TGU H994 P1 described in Straižys et al. (2016), hereafter Paper II. Colour indices with $\sigma=0.05-0.10 \mathrm{mag}$ (mostly those that include the ultraviolet magnitudes $U$ and $P$ ) are labelled with colons. The stars found to be binaries or those that have asymmetric images in CCD exposures were not classified into luminosity classes in the column of spectral types they are designated with double asterisks. 
Table 1. First five stars of the data catalogue in the VATT area measured in the Vilnius seven-colour system.

\begin{tabular}{|c|c|c|c|c|c|c|c|c|c|c|}
\hline No. & $\begin{array}{l}\text { RA }(\mathrm{J} 2000) \\
\mathrm{h} \mathrm{m} \mathrm{s}\end{array}$ & $\underset{\circ,{ }^{\prime \prime}}{\operatorname{Dec}(\mathrm{J} 2000)}$ & $\begin{array}{l}V \\
\text { mag }\end{array}$ & $\begin{array}{l}U-V \\
\text { mag }\end{array}$ & $\begin{array}{l}P-V \\
\text { mag }\end{array}$ & $\begin{array}{l}X-V \\
\text { mag }\end{array}$ & $\begin{array}{l}Y-V \\
\text { mag }\end{array}$ & $\begin{array}{l}Z-V \\
\text { mag }\end{array}$ & $\begin{array}{l}V-S \\
\text { mag }\end{array}$ & $\begin{array}{l}\text { Phot. } \\
\text { sp. type }\end{array}$ \\
\hline 1001 & $3: 58: 12.90$ & J & 18.002 & 4.963 & 4.314 & 2010 & J & 583 & 240 & $\mathrm{~K}$ \\
\hline 1002 & $3: 58: 13.76$ & $+53: 40: 56.3$ & 19.407 & & & 2.6 & 26 & 70 & 1.237 & $a-f$ \\
\hline 1003 & $3: 58: 14.23$ & $+53: 41: 19.9$ & 19.070 & & & 3.301 & 1.641 & 0.543 & 1.423 & $\mathrm{~g}$ \\
\hline 1004 & $3: 58: 14.41$ & $+53: 39: 16.4$ & 18.118 & 4.6 & 3.9 & 2.8 & 1. & 18 & 1.129 & $\mathrm{k} 2 \mathrm{IV}$ \\
\hline 1005 & $3: 58: 14.74$ & $+53: 35: 02.1$ & 17.043 & 6.341 & 5.615 & 3.973 & 1.794 & 0.678 & 1.620 & k2 III \\
\hline
\end{tabular}

Notes. The stars are numbered starting from 1001 to avoid confusion with the catalogue of Paper I. The running numbers, coordinates, $V$ magnitudes, six colour indices, and photometric spectral types are given. The full Table 1 for 302 stars is available at the CDS.

\section{Interstellar extinctions and distances based on Vilnius photometry}

For 628 stars from Paper I and for 238 stars from Table 1 with reliable spectral and luminosity classes, colour excesses $E_{X-V}$, $E_{Y-V}$, and $E_{V-S}$ were calculated as differences between the observed and intrinsic colour indices taken from Straižys (1992). Then the excesses $E_{X-V}$ and $E_{V-S}$ were transformed into $E_{Y-V}$ with the equations

$E_{Y-V}=0.58 E_{X-V}$

and

$E_{Y-V}=1.19 E_{V-S}$,

and the three values of $E_{Y-V}$ were averaged. The interstellar extinctions $A_{V}$ and distances $d$ (in pc) were calculated with the following equations:

$A_{V}=4.16 E_{Y-V}$

and

$\log d=0.2\left(V-M_{V}+5-A_{V}\right)$,

where $V$ are the observed magnitudes and $M_{V}$ are the magnitudes taken for a given spectral type from Straižys (1992). The errors of colour excesses, extinctions, and distances are described in Paper II. Typical errors of the extinction and distance are $\sigma\left(A_{V}\right) / A_{V}= \pm 0.06$, and $\sigma(d) / d= \pm 0.10$. The coefficient in Eq. (3) corresponds to the normal interstellar extinction law. The normality of the law is based on the analysis of colour excesses of B and A stars in the area TGU H994 P1 described in Paper II. We have also found that the slope of the reddening line in the $J-H$ vs. $H-K_{\mathrm{s}}$ diagram in both areas coincides. This means that the extinction law in the near-infrared is similar in both directions.

The plot $A_{V}$ vs. $d$ for 57 stars in the whole $1.5 \mathrm{deg}^{2}$ area with distances up to $250 \mathrm{pc}$ is shown in Fig. 2. All these stars are measured from the Moletai telescope exposures. A few stars in the same distance range measured from the VATT exposures are common in both catalogues. Two more possible K1-K1.5 dwarfs, Nos. 731 and 878 , with $A_{V}=3.6$ and $3.8 \mathrm{mag}$, respectively, fall into the same distance range, but their classification and distances are of lower accuracy, thus we do not plot these stars in Fig. 2.

To estimate distance to the nearest dust cloud, we applied the same method as in Paper II: the obscured (reddened) stars with the smallest distances were considered to be located at a distance of the front side of the dust cloud, but scattered to lower apparent distances because of negative errors. The main source of the distance error is the absolute magnitude of the star, which

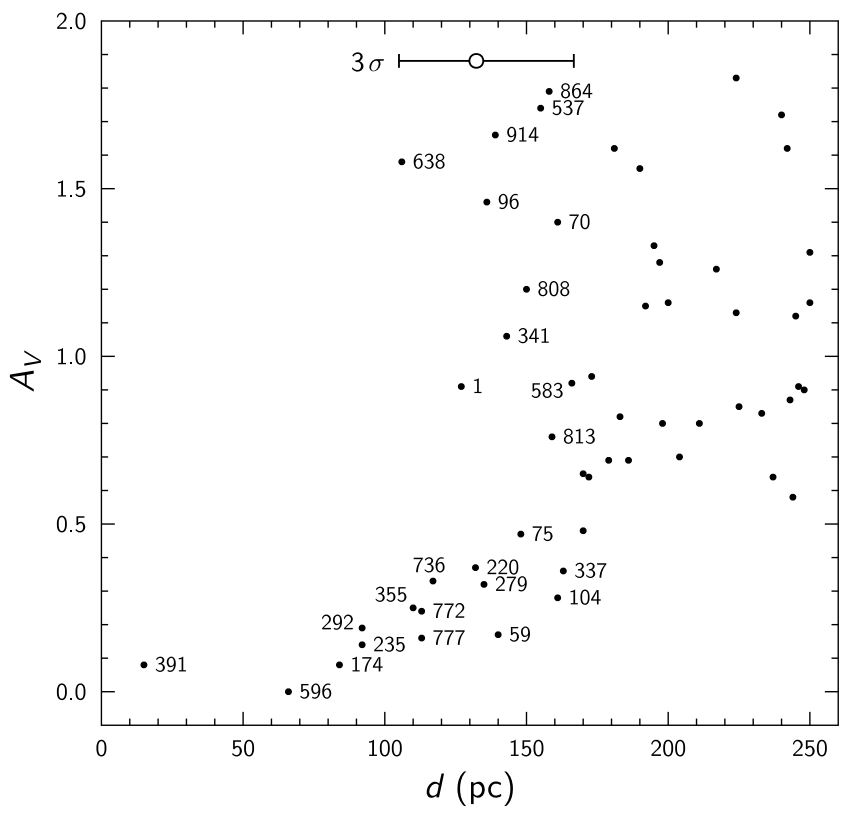

Fig. 2. Dependence of the extinction on distance for the investigated area up to $d=250 \mathrm{pc}$. The $3 \sigma$ error bar for $d=132 \mathrm{pc}$ with the extreme distances $105 \mathrm{pc}$ and $167 \mathrm{pc}$ is shown. The stars within this error bar are labelled with their numbers from Paper I.

is estimated from its spectral and luminosity classes. We accept that the $3 \sigma$ error of the absolute magnitude is $0.5 \mathrm{mag}$, and this corresponds to a scatter of distances by a factor of 1.26.

In Fig. 2 the nearest star with high extinction is No. 638 (g8.5 IV-V) at $105 \mathrm{pc}$. If this star has appeared at this apparent distance as a result of the $3 \sigma$ error of $d$, then its real distance should be $132 \mathrm{pc}$. This means that the distance to the nearest clouds in the area is close to $130 \mathrm{pc}$, that is, closer by $\sim 10 \mathrm{pc}$ than the nearby cloud chain TGU H994 P1 investigated in Paper II. However, we are not sure that all reddened stars within the error bar shown in Fig. 2 are at the same distance. As we noted in Paper II, the Camelopardalis clouds can have a considerable thickness along the sightline, which in the nearby Taurus complex is of the order of 25-30 pc (Loinard et al. 2011; Welsh et al. 2010; Lallement et al. 2014).

The surface distribution of the nearby reddened stars shows no relation to the surface density of the dust thermal emission across the Galaxy that is shown by the dust map from Schlegel et al. (1998) based on the IRAS and COBE/DIRBE $100 \mu \mathrm{m}$ observations. This means that the nearest dust layer at $\sim 130 \mathrm{pc}$ covers more or less the entire investigated area, and the dense clouds in the mentioned dust map are seen in the distant background. 


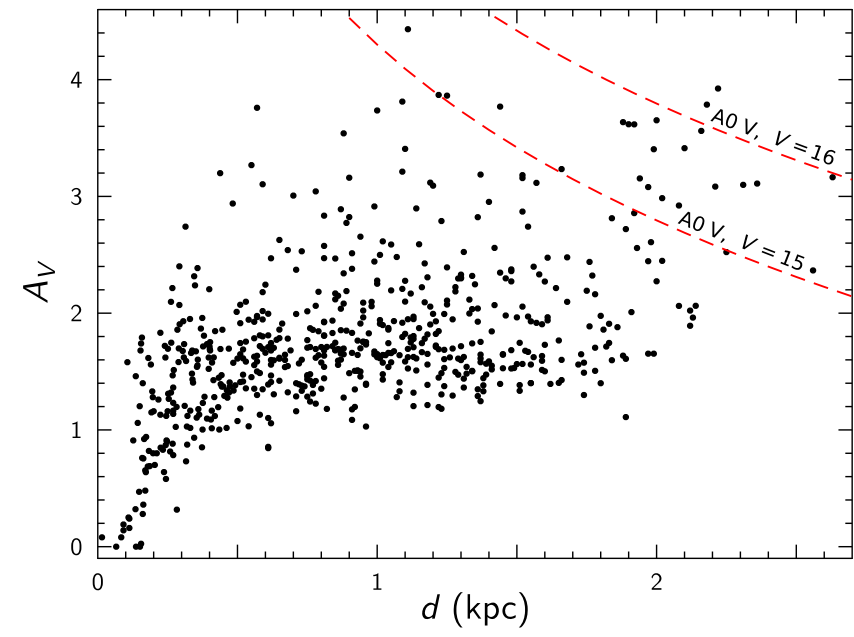

Fig. 3. Dependence of the extinction on distance for the investigated area up to $2.6 \mathrm{kpc}$ for 628 stars with two-dimensional classification, measured in the Moletai telescope exposures. The two red broken curves show the limiting magnitude effect for $\mathrm{A} 0 \mathrm{~V}$ at $V=15$ and $16 \mathrm{mag}$.

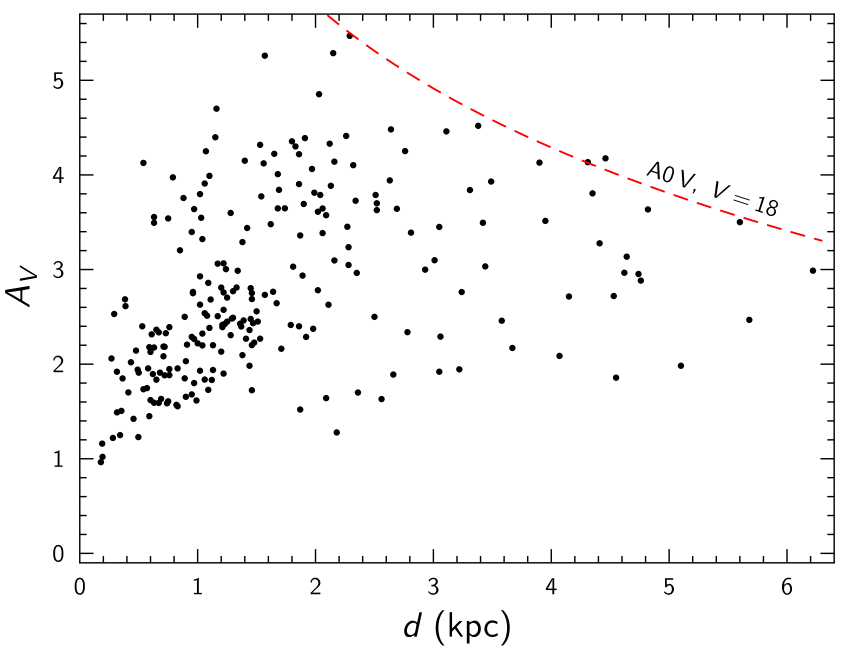

Fig. 4. Dependence of the extinction on distance for the investigated area up to $6 \mathrm{kpc}$ for 238 stars with two-dimensional classification, measured in the VATT telescope exposures. The red broken curve shows the limiting magnitude effect for A0 $\mathrm{V}$ at $V=18 \mathrm{mag}$.

The $A_{V}$ vs. $d$ plot up to $\sim 2.5 \mathrm{kpc}$ for the $1.5 \mathrm{deg}^{2}$ area is shown in Fig. 3 for 628 stars measured and classified from the Moletai telescope exposures. The extinction run with distance, starting from the jump at $130 \mathrm{pc}$, continues to rise quite steeply up to 200-300 pc. At this distance a considerable scatter of the extinction values begins. However, the majority of stars up to the Perseus Arm at $1.5 \mathrm{kpc}$ remain at the level $A_{V}=1.5-2.0 \mathrm{mag}$. The highest extinction is difficult to estimate since at high values of $A_{V}$ the selection effect that is due to the limiting magnitude starts to be effective, especially for A-F-G stars; see the limiting magnitude curves for A0 V stars. Behind the Perseus arm at $2.5 \mathrm{kpc}$ the stars are mostly fainter than $V=16 \mathrm{mag}$, and they were rejected because of the low accuracy of photometry and classification.

Figure 4 shows a similar plot up to $\sim 6 \mathrm{kpc}$ for 238 stars measured and classified in the smaller VATT area of intermediate transparency. The red broken curve in the right upper corner shows the limiting magnitude effect for the A0 V stars at $V=18 \mathrm{mag}$. The same curve is also valid for G5-K2 giants, which have absolute magnitudes similar to A0 V stars. Because of the fainter limiting magnitude, some stars are measured and classified up to 5-6 kpc distances. Moreover, much higher extinctions of up to $5 \mathrm{mag}$ and more become accessible. At 500-600 pc the extinction jump up to $A_{V}=3.5-4.0 \mathrm{mag}$ seems to be present. Some rise of the extinction is also possible in the Perseus Arm at $d>1.5 \mathrm{kpc}$. Three stars with $A_{V}$ close to 5 mag are observed within the Perseus arm, but the stars with stronger obscuration are absent because of the limiting magnitude effect. To better understand the extinction run at large distances, we must apply photometry in the infrared spectral range, which we do in the next section.

\section{Interstellar extinction based on 2 MASS and WISE photometry of red clump giants}

Another way to investigate interstellar extinction with distance is to use the red clump giants (RCGs), which have a low dispersion of their intrinsic colours and absolute magnitudes. In Paper II we proposed a method that allows identifying RCGs using the 2MASS and WISE near-infrared photometric systems. To identify RCGs we applied the following steps.

(1) First, we used the $J-H$ vs. $H-K_{\mathrm{s}}$ diagram to isolate stars lying within a reddening belt with a width $H-K_{\mathrm{s}}=0.1$ and a slope of $E_{J-H} / E_{H-K_{\mathrm{S}}} \approx 2.0$. The central line of this belt is the reddening line of RCGs, which starts at their intrinsic position with $J-H=0.46$ and $H-K_{\mathrm{s}}=0.09$ (Straižys \& Lazauskaitė 2009). This belt contains RCGs of different interstellar reddenings, but it also covers the sequence of normal RGB stars of spectral classes G5 to M5, as well as unreddened or weakly reddened dwarfs of spectral classes $\mathrm{K}$ and M0-M3. The width of this belt corresponds to the scatter of stars that is due to observational errors \pm 0.03 mag of $J, H$, and $K_{\mathrm{s}}$ magnitudes.

(2) Next, we formed a similar belt of RCGs in the two-colour diagram $H-W 2$ vs. $J-W 2$, where $J$ and $H$ are 2MASS magnitudes and $W 2$ is the magnitude of the WISE system with the mean wavelength at $4.6 \mu \mathrm{m}$. The width of this belt is $H-W 2=0.1$ and its central reddening line has the slope close to $E_{H-W 2} / E_{J-W 2}=0.51$ and the intrinsic point of RCGs is at $H-W 2=0.07$ and $J-W 2=0.56$. This diagram allows rejecting a considerable part of $\mathrm{G}-\mathrm{K}$ dwarfs and $\mathrm{M}$ giants, most of the asymptotic branch stars, and YSOs that contaminate the RCG belt in the $J-H$ vs. $H-K_{\mathrm{s}}$ diagram.

(3) Then we selected stars in common in the reddening belts of the two diagrams. The further rejection of unreddened and reddened dwarfs of spectral classes $\mathrm{G}-\mathrm{K}-\mathrm{M}$ and of subgiants of spectral classes $\mathrm{G}-\mathrm{K}$ was made with the use of the colour-magnitude diagram $K_{\mathrm{s}}$ vs. $H-K_{\mathrm{s}}$ as described in $\mathrm{Pa}-$ per II.

(4) The remaining RCG canditates were dereddened by the method described in Paper II and plotted in the intrinsic diagram $(J-H)_{0}$ vs. $\left(H-K_{\mathrm{s}}\right)_{0}$. To investigate interstellar extinction, we considered stars located only within the rectangle that is limited by the coordinates $(\mathrm{J}-\mathrm{H})_{0}$ between 0.42 and 0.54 , and $\left(H-K_{\mathrm{s}}\right)_{0}$ between 0.07 and 0.13 .

The equations used for the selection of RCGs and their dereddening are the same as in Paper II since the slopes of interstellar reddening lines in the present area do not differ from the area around TGU H994 P1. To select RCGs we used only stars whose errors of magnitudes were $\leq 0.03 \mathrm{mag}$ for $J$ and $K_{\mathrm{s}}$ and 
$\leq 0.04$ mag for $H$. We detected this decrease of accuracy for magnitudes $H$ in this area by intercomparing accuracies in different passbands.

After applying these procedures, the resulting list contains 714 suspected RCG stars. Despite the filtering, this list remains contaminated by a certain amount of normal G-K giants and subgiants of RGB as well as K-M dwarfs. Of these, the RGB giants are of little effect since they have similar values of the intrinsic colour indices and absolute magnitudes as the RCGs of the same spectral classes, which means that they yield similar extinctions and distances. We estimated the contamination percentage using 68 stars of the selected RCGs that have been observed and classified in the Vilnius system. Of these, $67 \%$ are confirmed to be red giants of spectral types G8-K2 III. The remaining $33 \%$ are dwarfs and subgiants. Fortunately, these stars are easy to recognize in the extinction versus distance plots, since they exhibit outstandingly low extinctions at large distances (if we accept that they are RCGs). This allowed us to identify and reject 27 additional dwarfs and subgiants.

Interstellar extinctions and distances of the selected RCGs were determined with Eqs. (18) and (19) of Paper II. Figure 5 shows the $A_{K_{\mathrm{s}}}$ vs. $d$ plot for 329 RCGs in the investigated area. The right-hand $y$-axis shows the extinctions transformed into the $A_{V}$ scale with the equation

$A_{V}=8.3 A_{K_{\mathrm{s}}}$,

which is valid for the normal interstellar extinction law. The relative errors of the extinction and distance are $\sigma\left(A_{K_{\mathrm{s}}}\right)= \pm 0.115 A_{K_{\mathrm{s}}}$ and $\sigma(d) / d= \pm 0.042$, see Straižys et al. (2015).

It is evident that in the present selection of RCGs the extinctions $A_{K_{\mathrm{s}}}$ up to $1.0 \mathrm{mag}$ are observed, and this corresponds to $A_{V} \approx 8.5 \mathrm{mag}$. The extinction run with distance can only be followed at $d>1 \mathrm{kpc}$ since at smaller distances the identified RCGs are rather scarce because their images are saturated in the WISE $W 2$ passband. The highest extinction $A_{V}$ in the Local Arm is close to 4 mag, which means that it agrees with Figs. 3 and 4. In Fig. 5, at the beginning of the Perseus Arm, $d>1.5 \mathrm{kpc}$, we observe a considerable increase in the number of stars with the extinction $A_{K_{\mathrm{s}}}>0.6 \mathrm{mag}$. These stars are probably located behind the dense dust clouds of the Local and/or the Perseus arms.

In Fig. 6 we show the surface distribution of RCGs plotted as circles of different diameters proportional to their extinctions. The background shows the surface density of dust across the Galaxy from Schlegel et al. $(1998)^{2}$. It is evident that RCGs are lacking in the densest parts of P7 and P8 where the Perseus Arm objects are not seen because of high extinction in the Local Arm.

At the same time, the lowest $A_{V}$ extinction in the Perseus Arm remains at about 2 mag, which is higher by only $\sim 0.5 \mathrm{mag}$ than in the Local Arm (Fig. 3). These RCGs are probably seen through transparent spaces between the clouds above (or below) the dust layer of the Perseus Arm. The apparent decrease in the extinction at $d>3 \mathrm{kpc}$ is a result of the limiting magnitude in the passbands with the longest wavelengths. For the $K_{\mathrm{s}}$ passband this corresponds to the apparent magnitude $12-12.5$. For the $W 2$ passband the limiting magnitude is very similar since the absolute magnitudes of RCGs in both $K_{\mathrm{s}}$ and $W 2$ almost coincide.

\section{Identification of YSOs and a new infrared cluster}

The YSOs in the area were identified using the Koenig \& Leisawitz (2014) classification scheme, which

\footnotetext{
2 In Fig. 6 positions of the Dobashi et al. (2005) clumps P7 and P8 are slightly shifted to the nearest intensity maxima of the dust emission shown in the dust map of Schlegel et al. (1998).
}

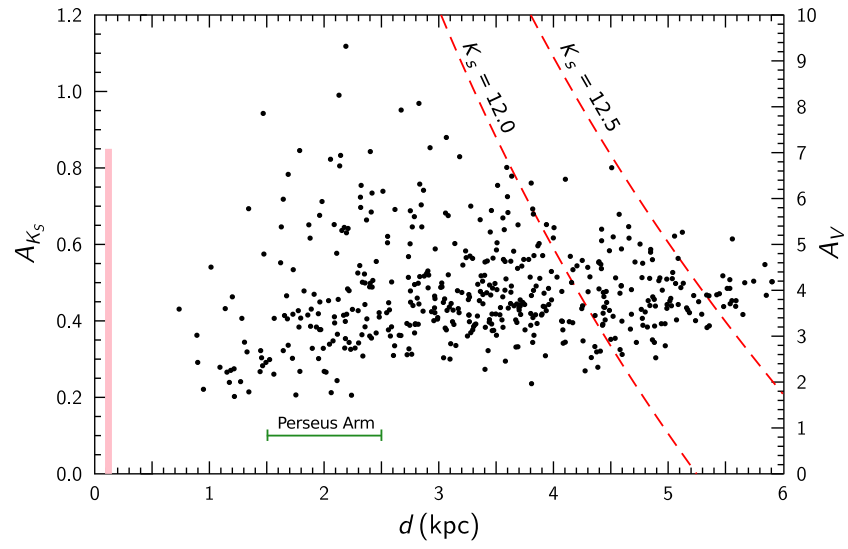

Fig. 5. Extinction $A_{K_{\mathrm{S}}}$ vs. distance in the investigated area for 662 RCGs identified by combining the 2MASS and WISE magnitudes. The vertical line designates the accepted position of the nearest layer of dust at $130 \mathrm{pc}$. The two broken curves designate RCGs at the limiting magnitudes $K_{\mathrm{s}}=12$ and 12.5. The distance of the Perseus Arm is the average value from Reid et al. (2014) and Vallée (2014).

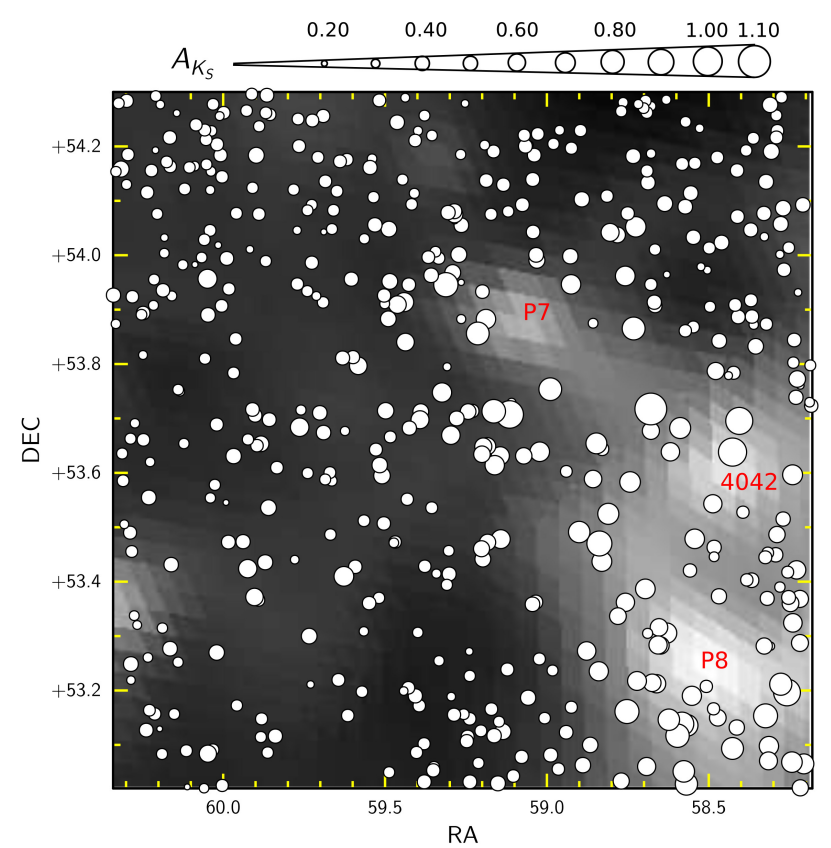

Fig. 6. Surface distribution of the extinction $A_{K_{\mathrm{S}}}$ for RCGs. Diameters of the circles are proportional to their extinction values. The dust map from Schlegel et al. (1998) is shown in the background. The brightest areas correspond to an across-the-Galaxy extinction $A_{K_{\mathrm{S}}}$ between 1.2-1.8 mag, while in the darkest areas the extinction is $\sim 0.4 \mathrm{mag}$.

combines the WISE and 2MASS near- and mid-infrared colours. In the investigated area we found 88 YSOs listed in Table 2, 25 of which belong to class I and 63 to class II. Their surface distribution, shown in Fig. 7, exhibits strong clustering into the dust clouds seen in the dust map by Schlegel et al. (1998). Only a few objects are scattered around the dark clouds. The largest number of YSOs is concentrated in dust clump P7 and the infrared cluster FSR 655 with the coordinates $\mathrm{RA}=03: 56: 14, \mathrm{Dec}=+53: 51: 54$ (Froebrich et al. 2007). Three of these YSOs were identified earlier by Straižys \& Laugalys (2007b) and about 16 by Romero \& Cappa (2009) using the 2MASS, MSX and IRAS surveys. 
Table 2. First five YSOs identified in the investigated area with the method described by Koenig \& Leisawitz (2014).

\begin{tabular}{rcrrrrrrr}
\hline \hline No. & WISE & $\begin{array}{r}W 1 \\
\mathrm{mag}\end{array}$ & $\begin{array}{r}W 2 \\
\mathrm{mag}\end{array}$ & $\begin{array}{r}W 3 \\
\mathrm{mag}\end{array}$ & $\begin{array}{r}J \\
\mathrm{mag}\end{array}$ & $\begin{array}{r}H \\
\mathrm{mag}\end{array}$ & $\begin{array}{r}K_{\mathrm{s}} \\
\mathrm{mag}\end{array}$ & Type \\
\hline 1 & $\mathrm{~J} 035252.53+532344.7$ & 11.866 & 10.921 & 8.361 & 15.120 & 13.865 & 13.038 & YSO2 \\
2 & $\mathrm{~J} 035252.54+532349.7$ & 12.183 & 11.696 & 9.457 & 14.585 & 13.292 & 12.767 & YSO2 \\
3 & $\mathrm{~J} 035303.05+531554.4$ & 12.953 & 12.457 & 10.119 & 15.155 & 14.128 & 13.565 & YSO2 \\
4 & $\mathrm{~J} 035309.07+531612.9$ & 11.865 & 11.244 & 9.571 & 15.202 & 13.817 & 12.725 & YSO2 \\
5 & $\mathrm{~J} 035314.27+531933.2$ & 12.415 & 11.189 & 8.366 & 16.387 & 15.234 & 14.093 & YSO1 \\
\hline
\end{tabular}

Notes. The full Table 2 is available at the CDS.

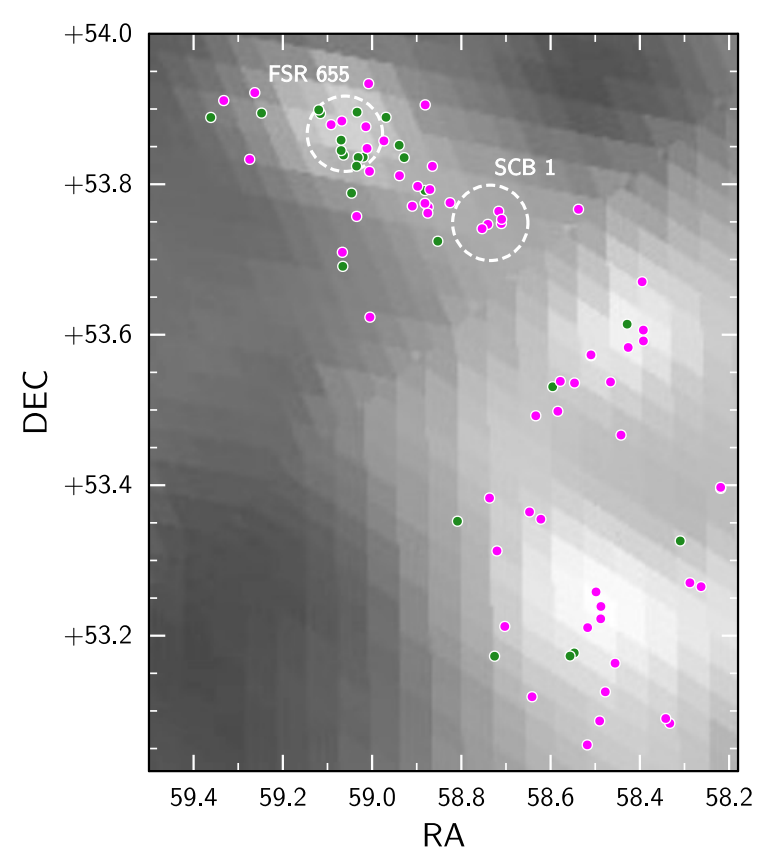

Fig. 7. Surface distribution of YSOs of types I (green circles) and II (pink circles) in the investigated area. The dust map from Schlegel et al. (1998) is shown in the background. The cluster FSR 655 and the new possible infrared cluster SCB 1 are marked with two broken circles of $6^{\prime}$ diameters.

A compact group of five YSOs is located at RA = 03:54:56, Dec $=+53: 45$. These stars are probably members of a small heavily reddened cluster with an angular diameter of 2.0-2.5 arcmin. Hereafter we call this possible cluster SCB 1. Within the mentioned diameter, about 30 stars down to $K_{\mathrm{s}} \approx$ 15 mag can be counted (Fig. 8). We cannot determine which stars are members of this possible cluster and which are background stars. The extinction of stars in this cluster probably originates in the local dust layer at $130-300 \mathrm{pc}$ and the dust cloud TGU H942 P7. The cluster almost coincides with the dark cloud Dobashi 4040 (Dobashi 2011). From a statistical analysis of $J-H$ and $H-K_{\mathrm{s}}$ colours in this cloud, Dobashi (2011) found its average extinction to be $A_{V}=6.85 \mathrm{mag}$. For the extinction across the Galaxy, the dust map calibrated by Schlafly \& Finkbeiner $(2011)^{3}$ in this direction gives a value of $A_{V}=7.28 \mathrm{mag}$.

\section{Discussion}

The investigated area is rather non-uniform with respect to distribution of interstellar extinction in the Local Arm. The highest extinction is found in the directions of clumps P7 and P8,

\footnotetext{
3 The NASA/IPAC Extragalactic Database, NED.
}

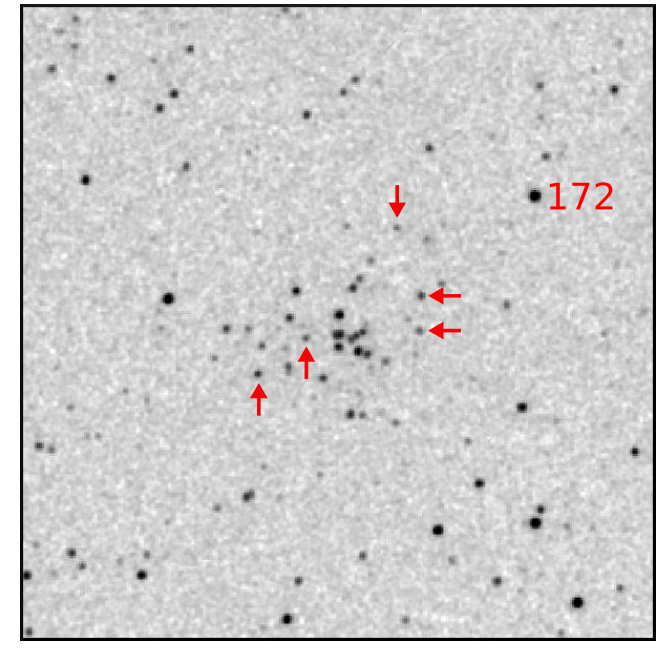

Fig. 8. New possible infrared cluster SCB 1 at $\mathrm{RA}=03: 54: 56$, Dec $=$ $+53: 45$ identified in the investigated area. The image of $0.1 \times 0.1 \mathrm{deg}^{2}$ size in the 2MASS filter $K_{\mathrm{s}}$ is taken from the SkyView virtual observatory. The five YSOs are marked with arrows. Star 172 is present in the catalogue of Paper I ( $V=12.53$, A5 V).

including the chain of the Dobashi (2011) clouds with numbers 4040-4051. However, differences in the extinction become evident only at distances exceeding $\sim 400$ pc. At smaller distances the whole area is covered more or less uniformly by the nearest clouds. The nearest cloud layer begins at a distance of $130 \pm 10 \mathrm{pc}$, where the extinction $A_{V}$ steeply rises from 0.3 to $1.8 \mathrm{mag}$ (Fig. 2). Between 200 and $400 \mathrm{pc}$ the extinction continues to rise up to $2.7 \mathrm{mag}$. Another jump of extinction up to $\sim 4$ mag is possible at a distance of $500-600 \mathrm{pc}$, this is better visible in Fig. 4.

The space between the Local and Perseus arms (1-1.5 kpc) is expected be free of dust. This, however, seems only to be confirmed by RCGs in Fig. 5 since the extinction in Figs. 3 and 4 in this distance range is affected by the limiting magnitude effect and the scatter of stars caused by classification and distance errors. For some RCGs that are located within and behind the Perseus Arm, the extinction $A_{V}$ becomes as high as $\sim 8$ mag (Fig. 5). However, these stars lie outside the densest parts of the clouds. Within the clouds the extinction can be estimated by the dust maps by Schlegel et al. (1998) based on the $100 \mu \mathrm{m}$ dust emission, calibrated by Schlafly \& Finkbeiner (2011), see the NED database. These maps give the values of $A_{V} \sim 9.3 \mathrm{mag}$ in the centre of P7 (or Dobashi 4043), 15.0 mag between Sh2205 and clump P8, and 13.4 mag in the dust cloud Dobashi 4042 above Sh2-205 (see Fig. 1).

The lowest values of $A_{V}$ at distances $>1 \mathrm{kpc}$, determined from Figs. $3-5$, are close to $1.5-2.0 \mathrm{mag}$. In these directions 
distant stars are probably observed through semi-transparent windows between clouds.

The extinction distribution in the area can be compared with the distribution of molecular clouds by applying radio observations of CO lines from Dame et al. (2001). In this case, instead of the distance, we may use radial velocities of molecular clouds, which are functions of the distance due to Galactic rotation. The positions of $\mathrm{CO}$ clouds within our area, shown in Straižys \& Laugalys (2007a, 2008), approximately coincide with the highest dust concentrations. The analysis of the data reported by Dame et al. (2001) shows that the local standard of rest (LSR) radial velocities of CO clouds P7 and P8 are mostly concentrated in the three intervals with maxima at $-2.5 \mathrm{~km} \mathrm{~s}^{-1}$, $\sim-6 \mathrm{~km} \mathrm{~s}^{-1}$, and $\sim-34 \mathrm{~km} \mathrm{~s}^{-1}$. The first two intervals correspond to the Local Arm, the third one to the Perseus Arm. To estimate galactocentric distances $R$ to $\mathrm{CO}$ clouds, we applied the equation of kinematic distances:

$R=R_{0} \sin (\ell) \frac{V_{R}}{V+V_{0} \sin (\ell)}$,

where $V$ is its measured radial velocity, $V_{R}$ is the accepted galactocentric orbital velocity of the cloud (taken from the Galactic rotation curve), $R_{0}$ is the galactocentric radius of the solar orbit, $V_{0}$ is the solar orbital velocity, and $\ell$ is the Galactic longitude (in our case $148.3^{\circ}$ ). In the calculations we took the values of $R_{0}$ and $V_{0}$ from Bovy et al. (2012) and Reid et al. (2014), in both cases the flat Galactic rotation curve was accepted.

Heliocentric distances to the clouds $d$ were calculated from $R$ with the equation

$d=\frac{\left(R-R_{0}\right)}{\cos (180-\ell)}$.

For the parameters of Bovy et al. (2012), $R_{0}=8.10 \mathrm{kpc}$ and $V_{0}=218 \mathrm{~km} \mathrm{~s}^{-1}$, we obtain $d=208 \mathrm{pc}$ at $V_{r}=-2.5 \mathrm{~km} \mathrm{~s}^{-1}$ and $d=520 \mathrm{pc}$ at $V_{r}=-6 \mathrm{~km} \mathrm{~s}^{-1}$. For the parameters of Reid et al. (2014), $R_{0}=8.34 \mathrm{kpc}$ and $V_{0}=240 \mathrm{~km} \mathrm{~s}^{-1}$, the corresponding distances $d$ are $196 \mathrm{pc}$ and $485 \mathrm{pc}$. Thus, the first layer of CO clouds with the kinematic distance $\sim 200$ pc can be related to the local dust layer between 130 and 300 pc seen in Figs. 3 and 4. As we have noted in Paper II, this layer is a part of the large Camelopardalis dust cloud system that is similar to the Taurus clouds, located on the opposite side of the Galactic equator, which extends up to the Lindblad Ring and includes the Per OB3 association and the Cas-Tau moving group of B-type stars. The second CO layer with the kinematic distance $\sim 500 \mathrm{pc}$ can correspond to the jump of extinction at 500-600 pc described in Sect. 3 .

Since the infrared cluster FSR 655 and the new cluster SCB 1, identified in this paper, seem to be related with the chain of dust clouds P7, Dobashi 4042, and P8 and with the concentration of YSOs in these clouds, their distances are expected to be similar, probably within 500-600 pc.

We do not give any kinematic distance corresponding to the velocity $-34 \mathrm{~km} \mathrm{~s}^{-1}$ because $\mathrm{CO}$ clouds and star-forming regions in the Perseus Arm considerably depart from the circular rotation that is due to streaming and expanding motions (Sitnik 2003; Vallée 2008; Choi et al. 2014; Foster \& Brunt 2015).

The distance to the Sh2-205 nebula is of a special interest. Its ionised areas seem to be tangled with the dust clump P8, which leaves the impression that both the nebula and the dust cloud are at the same distance. Trying to verify this, we collected from the literature all known determinations of radial velocities of the nebula and the adjacent H I and CO clouds to calculate their kinematic distances. Unfortunately, the results described in the next paragraph are contradictory.

The first value of the radial velocity of Sh2-205, $-25.8 \mathrm{~km} \mathrm{~s}^{-1}$, corresponding to its $\mathrm{H} \alpha$ line, was published by Blitz et al. (1982). However, in the same paper a note is given that another $\mathrm{H} \alpha$ line is present at $-7 \mathrm{~km} \mathrm{~s}^{-1}$, which is considered to belong to the foreground. For the $\mathrm{H} \alpha$ line, Fich et al. (1990) found a value of $-16.8 \mathrm{~km} \mathrm{~s}^{-1}$. Fich \& Blitz (1984) for the associated CO cloud found a value of $-25.8 \mathrm{~km} \mathrm{~s}^{-1}$. Foster \& MacWilliams (2006) found for the associated HI cloud $-6.0 \mathrm{~km} \mathrm{~s}^{-1}$. Foster \& Brunt (2015) found $-6.48 \mathrm{~km} \mathrm{~s}^{-1}$ for $\mathrm{CO}$ and $-14.24 \mathrm{~km} \mathrm{~s}^{-1}$ for H I. The last value of velocity for $\mathrm{CO}$ is very close to the middle value obtained from the CO data of Dame et al. (2001) that we quoted above in this section. The values close to $-20 \mathrm{~km} \mathrm{~s}^{-1}$ are more appropriate to the Perseus Arm. To explain these differences in the measured radial velocities, we may suggest peculiar motions at different positions of the clouds. In some cases, the velocities might be measured for the clouds of the Perseus Arm seen through translucent clouds in the vicinity of Sh2-205. A dedicated investigation of the velocity field in the vicinity of this nebula would be helpful.

In the Introduction, we mentioned the possible ionising stars of the Sh2-205 nebula: HD 24431, (O9 from III to V), HD 24094 (CY Cam, B1 III to V), HD 23675 (B0 III), and ALS 7793 (B1 V). They are all located within $1^{\circ}$ from Sh2-205, but only two of them, CY Cam and ALS 7793, are within the investigated area. The $\mathrm{H} \alpha$ map from Finkbeiner (2003), available at the SkyView Virtual Observatory, shows that the first two of these stars are surrounded by dense H II regions. The northern region around CY Cam is the Sh2-205 nebula; it is located at the right-hand edge of the area investigated in the present paper. The southern region around HD 24431 is located about $0.5^{\circ}$ south of the lower edge of our area. The IRAS images and the composite WISE map ${ }^{4}$ show that in $20-60 \mu \mathrm{m}$ maps CY Cam is surrounded by a double lens-like $4^{\prime} \times 7^{\prime}$ spot of emission that probably originates in warm dust grains. This emission is absent from the IRAS $100 \mu \mathrm{m}$ map and in the submillimeter Planck bands. At an angular distance of $12-15^{\prime}$ this lens is surrounded by an envelope of rectangular shape with emission originating in cold dust grains (IRAS $100 \mu \mathrm{m}$ map) and the molecules of polycyclic aromatic hydrocarbons (PAHs, WISE $12 \mu \mathrm{m}$ map). In the vicinity of ALS 7793, both the dust and PAH emissions are also present. The star HD 23675 does not exhibit its own H II region nor emission from dust and PAH molecules.

The photometric distance to CY Cam is uncertain because of its doubtful luminosity class and because the star might be a close binary. If the star is of spectral class $\mathrm{B} 1 \mathrm{~V}$ with $M_{V}=-3.4 \mathrm{mag}$, its extinction is $A_{V}=2.25 \mathrm{mag}$ and the distance is $d=780 \mathrm{pc}$. If the star is a binary with two identical components, then its distance needs to be increased by a factor of 1.41 , meaning that it becomes about $1.1 \mathrm{kpc}$. This would mean that the star and the Sh2-205 nebula are much farther from the Sun than the chain of dust clouds scattered in its apparent vicinity. Some values of radial velocities of the nebula, listed above in this section, agree with a possible location in the Perseus Arm.

\section{Conclusions}

We have studied interstellar extinction in three dimensions in the direction of two clumps (P7 and P8) of the dust cloud

\footnotetext{
4 WISE multimedia gallery images, wise.ssl.berkeley.edu/ gallery_images.html
} 
TGU H942 that is located in the vicinity of the emission nebula Sh2-205 near the border between the Camelopardalis and Perseus constellations. For this, we used the results of our CCD photometry in the Vilnius system and photometric classification of 628 stars in a $1.5 \mathrm{deg}^{2}$ area down to $V=16$ mag and 238 stars in a $0.04 \mathrm{deg}^{2}$ area down to $\sim 19 \mathrm{mag}$. Additionally, to investigate the extinction, we applied 662 RCGs that have been identified with infrared photometry from the 2MASS and WISE surveys. The resulting extinction versus distance plots were compared with radio observations of the molecular $\mathrm{CO}$ emission from Dame et al. (2001) and the dust distribution across the Galaxy from Schlegel et al. (1998), based on the $100 \mu \mathrm{m}$ observations with the IRAS and COBE orbiting observatories. Although distances to the dust layers in the extinction versus distance diagrams are not of high accuracy, they are strengthened by additional information on radial velocities of $\mathrm{CO}$ clouds combined with the Galactic rotation curve.

The surface distribution of interstellar extinction in the area is nonuniform, exhibiting the highest values of obscuration in the right-hand and upper parts of the area, where the emission nebula Sh2-205 and dust clumps P7 and P8 are located. We conclude that at least three layers of dust and $\mathrm{CO}$ molecules are present in the direction we investigated. The first layer with $A_{V} \approx 1.8 \mathrm{mag}$ begins at about $130 \mathrm{pc}$ and covers more or less the whole area. This layer has a considerable depth, and in it a continuous rise of $A_{V}$ up to $2.5 \mathrm{mag}$ at $\sim 400 \mathrm{pc}$ is observed. At about $500-600 \mathrm{pc}$ another jump of $A_{V}$ up to $3.5-4.0 \mathrm{mag}$ is possible. Clumps P7 and P8 are located in this dust layer. However, we cannot conclude about the distance of the emission nebula Sh2-205, which can belong to the Perseus Arm. The third extinction rise is related to the Perseus Arm at $1.5-2.5 \mathrm{kpc}$ from the Sun. All these layers, especially the 500-600 pc layer and the Perseus Arm layer, are spotted, exhibiting many semitransparent windows where $A_{V}$ does not exceed 1.5-2.0 mag. The most transparent is the upper part of the area with declination $>54.0^{\circ}$. At the same time, the extinction $A_{V}$ across the Galaxy in the densest centres of the clouds P7 and Dobashi 4042 is as high as 13-15 mag.

Dust clouds in the 500-600 pc layer exhibit strong starforming activity; we identified 25 YSOs of class I (with envelopes) and 63 YSOs of class II (with discs). About 12 YSOs are concentrated in one of the known infrared clusters, FSR 655, and five YSOs lie in the cluster SCB 1 that we discovered in this investigation.

Acknowledgements. The use of the Simbad (CDS), NED (NASA), SkyView (NASA) and CO Data Archive (Smithsonian Astrophysical Observatory Radio Telescope Data Center) databases is acknowledged. We are grateful to Viktor Toth for important remarks.

\section{References}

Avedisova, V. S., \& Kondratenko, G. I. 1984, Nauchnye Informatsii, 56, 59 Blitz, L., Fich, M., \& Stark, A. A. 1982, ApJS, 49, 183 Bouigue, R. 1959, Publ. Obs. Haute Provence, 4, 52

Bovy, J., Prieto, C. A., Beers, T. C., et al. 2012, ApJ, 759, 131

Čepas, V., Zdanavičius, J., Zdanavičius, K., et al. 2013, Balt. Astron., 22, 223, Paper I

Choi, Y. K., Hachisuka, K., Reid, M. J., et al. 2014, ApJ, 790, 99

Conti, P. S., \& Alschuler, W. R. 1971, ApJ, 170, 325

Dame, T. M., Hartmann, D., \& Thaddeus, P. 2001, ApJ, 547, 792

De Cat, P., Briquet, M., Aerts, C., et al. 2007, A\&A, 463, 243

Dobashi, K. 2011, PASJ, 63, S1

Dobashi, K., Uehara, H., Kandori, R., et al. 2005, PASJ, 57, S1

Duerbeck, H. W. 1997, IBVS, 4513, 1

Fich, M., \& Blitz, L. 1984, ApJ, 279, 125

Fich, M., Treffers, R. R., \& Dahl, G. P. 1990, AJ, 99, 622

Finkbeiner, D. P. 2003, ApJS, 146, 407

Foster, T., \& Brunt, C. M. 2015, AJ, 150, 147

Foster, T., \& MacWilliams, J. 2006, ApJ, 644, 214

Froebrich, D., Scholz, A., \& Raftery, C. I. 2007, MNRAS, 374, 399

Gaze, V. F., \& Shain, G. A. 1955, Izv. Crimean Astrophys. Obs., 15, 11

Johnson, H. M. 1956, ApJ, 124, 90

Koenig, X. P., \& Leisawitz, D. T. 2014, ApJ, 791, 131

Lallement, R., Vergely, J.-L., Valette, B., et al. 2014, A\&A, 561, A91

Laugalys, V., Kazlauskas, A., Boyle, R. P., et al. 2004, Balt. Astron., 13, 1

Loinard, L., Mioduszewski, A. J., Torres, R. M., et al. 2011, in Rev. Mex. Astron. Astrofis. Conf. Ser., 40, 205

Morgan, W. W., Whitford, A. E., \& Code, A. D. 1953, ApJ, 118, 318

Reid, M. J., Menten, K. M., Brunthaler, A., et al. 2014, ApJ, 783, 130

Roeser, S., Demleitner, M., \& Schilbach, E. 2010, AJ, 139, 2440

Romero, G. A., \& Cappa, C. E. 2008, MNRAS, 387, 1080

Romero, G. A., \& Cappa, C. E. 2009, MNRAS, 395, 2095

Rydström, B. A. 1978, A\&AS, 32, 25

Schlafly, E. F., \& Finkbeiner, D. P. 2011, ApJ, 737, 103

Schlegel, D. J., Finkbeiner, D. P., \& Davis, M. 1998, ApJ, 500, 525

Sharpless, S. 1959, ApJS, 4, 257

Sitnik, T. G. 2003, Astron. Lett., 29, 311

Sota, A., Maíz Apellániz, J., Walborn, N. R., et al. 2011, ApJS, 193, 24

Straižys, V. 1992, Multicolor Stellar Photometry (Tucson, Arizona: Pachart Pub-

lishing House), http: //www.itpa.1t/MulticolorStellarPhotometry/

Straižys, V., \& Laugalys, V. 2007a, Balt. Astron., 16, 167

Straižys, V., \& Laugalys, V. 2007b, Balt. Astron., 16, 327

Straižys, V., \& Laugalys, V. 2008, in Handbook of Star Forming Regions, Vol. 1, ed. B. Reipurth (ASP), 294

Straižys, V., \& Lazauskaitè, R. 2009, Baltic Astron., 18, 19

Straižys, V., Vrba, F. J., Boyle, R. P., et al. 2015, AJ, 149, 161

Straižys, V., Čepas, V., Boyle, R. P., et al. 2016, A\&A, 585, A31 (Paper II)

Vallée, J. P. 2008, AJ, 135, 1301

Vallée, J. P. 2014, AJ, 148, 5

Welsh, B. Y., Lallement, R., Vergely, J.-L., \& Raimond, S. 2010, A\&A, 510, A54

Zdanavičius, J., \& Zdanavičius, K. 2002, Balt. Astron., 11, 441

Zdanavičius, J., Černis, K., Zdanavičius, K., \& Straižys, V. 2001, Balt. Astron., 10,349

Zdanavičius, K., Straižys, V., Zdanavičius, J., et al. 2012, A\&A, 544, A49 